\title{
Hexagonal To Orthorhombic Symmetry Reduction In a Lamellar Ti-42Al-8.5Nb Alloy
}

\author{
Heike Gabrisch ${ }^{1}$, Uwe Lorenz ${ }^{2}$, Florian Pyczak ${ }^{3}$, Marcus Rackel ${ }^{4}$, Andreas Stark ${ }^{5}$ \\ ${ }^{1 .-5 .}$ Helmholtz-Zentrum Geesthacht, Institute of Materials Research, Geesthacht, Germany.
}

Intermetallic TiAl-alloys are attractive candidates for structural applications in aero-engines because of their excellent high-temperature strength, low density and good corrosion resistance. A general drawback of intermetallic compounds is their low ductility. Alloying with niobium seems to improve the alloys' properties. For example the addition of 5-10 at.- $\%$ of $\mathrm{Nb}$ to the alloy Ti-(40-44)Al increased high temperature yield stress and room temperature ductility compared to its binary counterpart. These improvements were linked to the presence of an orthorhombic phase in the alloy's microstructure [1]. Motivated by the prospects for alloy design we studied the formation, thermal stability and the spatial arrangement of orthorhombic phases in an alloy of comparable composition. We chose a powder metallurgical route to produce the alloy $\mathrm{Ti}-42 \mathrm{Al}-8.5 \mathrm{Nb}$ and optimized heat treatments to promote the formation of orthorhombic phases, details are published in [2, 3]. The alloy investigated here was compacted by hot iso-static pressing (HIP) at $1250^{\circ} \mathrm{C}$ and $200 \mathrm{MPa}$ and then annealed at $650^{\circ} \mathrm{C}$ for 2 hours followed by oil quench.

In the HIPed condition the alloy consists of the major phases $\alpha_{2}$ (hexagonal, D0 ${ }_{19}$ ) and $\gamma$ (tetragonal,L10) that form lamellar colonies surrounded by seams of $\gamma$ phase and a few small wo grains, Fig. 1a. This general morphology does not change during subsequent annealing since transformations are observed in the $\alpha_{2}$ phase only. The alignment of $\alpha_{2}$ and $\gamma$ phases within the lamellar colonies is $(0001)_{\alpha 2} / /\{111\}_{\gamma}$ and $\langle 1-210\rangle_{\alpha 2} / /\langle 110\rangle_{\gamma}$ [4]. The nature of the orthorhombic phase in the annealed alloy as being O-phase $\left(\mathrm{TiAlNb}_{2}, \mathrm{Cmcm}\right.$ symmetry) was confirmed by single crystal selected area diffraction and by highenergy-X-ray diffraction. Dark field imaging along the $\alpha_{2}$ [10-10] direction parallel to the $\alpha_{2} / \gamma$ interfaces is used to determine that the phase is located within $\alpha_{2}$ laths of lamellar $\left(\alpha_{2}+\gamma\right)$ colonies (Fig. 1b, c). At higher magnification in Fig. 2a periodic contrast changes are observed within $\alpha_{2}$ comparable to the contrast behavior at low magnification in Fig. 1b. A comparison of image contrast and FFT of the small insets shown in Fig. 2a to simulated diffraction and high resolution images allows to identify the two stripes as differently oriented regions of O-phase. For comparison in $\alpha_{2}$ [0001] projection regions of hexagonal and two fold symmetry corresponding to $\alpha_{2}$ and orthorhombic B19 phase can be distinguished within former $\alpha_{2}$ laths. Imaging along the [0001] direction in STEM mode shows faceting within the former $\alpha_{2}$ lamellae, Fig. 2b. The nature of these facets is not understood so far.

Summarizing our observations we have identified a $\mathrm{Nb}$ depleted form of the orthorhombic O-phase for the first time. The O-phase has the ideal composition $\mathrm{Ti}_{2} \mathrm{AlNb}$ and has been reported previously for ternary $\mathrm{Ti}-\mathrm{Al}-\mathrm{Nb}$ alloys with low $\mathrm{Al}$ and high $\mathrm{Nb}$ content in the composition range Ti-(12-31)Al-(12.537) $\mathrm{Nb}$ [5]. Due to the low $\mathrm{Nb}$ content in the present alloy characteristic ordering of $\mathrm{Nb}$ to specific lattice sites in the $\mathrm{Cmcm}$ lattice was not observed. However the lattice distortion within the $\alpha_{2}$ basal plane resulting from the hexagonal to orthorhombic transition is visible in form of angular distortions in the hexagonal basal plane and in nearby zone axes. From high resolution imaging some amounts of the orthorhombic B19 phase cannot be excluded, even though no more than four stable phases are expected to exist in the system at a given temperature according to Gibb's phase rule. [6] 


\section{References:}

[1] F Appel, M Oehring, J. Paul, Advanced Engineering Materials 8 (2006), p. 371.

[2] R. Gerling, H. Clemens, and F.P. Schimansky, Advanced Engineering Materials 6 (2004), p.23.

[3] M. Rackel et al, MRS Fall 2014: 10.1557/opl.2015.31.

[4] M.J. Blackburn, Science, Technology, and Application of Titanium (1970), p. 633.

[5] K. Muraleedharan et al, Intermetallics 3 (1995), p. 187.

[6] The authors thank F.P. Schimansky for the preparation of the powder used in this alloy.

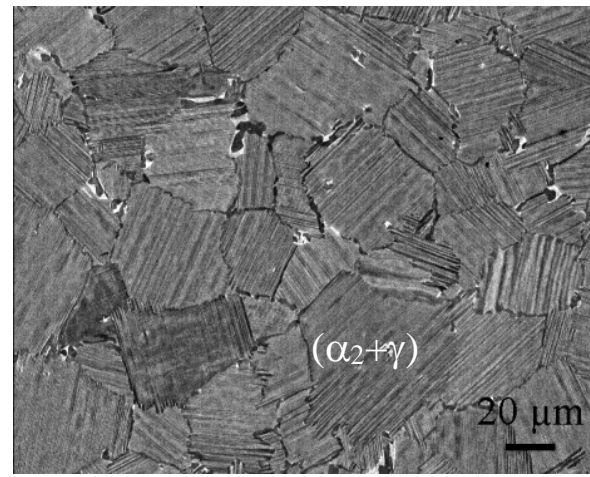

a.

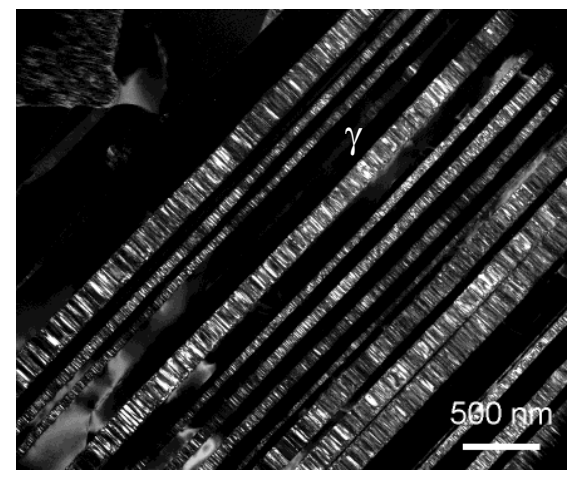

b.

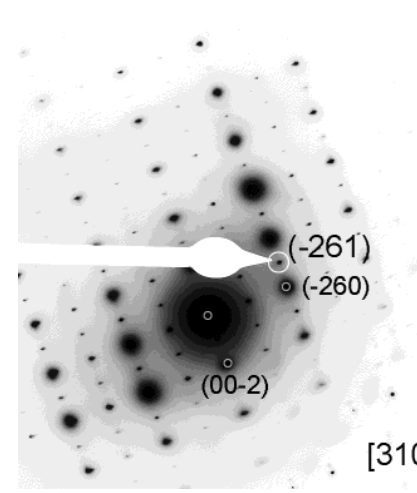

c.

Figure 1. a. Overview showing lamellar $\left(\alpha_{2}+\gamma\right)$ colonies by SEM imaging b. Dark field image taken with (-261) reflection of O-phase illustrating the presence of orthorhombic phase within $\alpha_{2}$ laths $\mathbf{c}$. Diffraction condition used for dark field imaging (indexing according to O-phase).

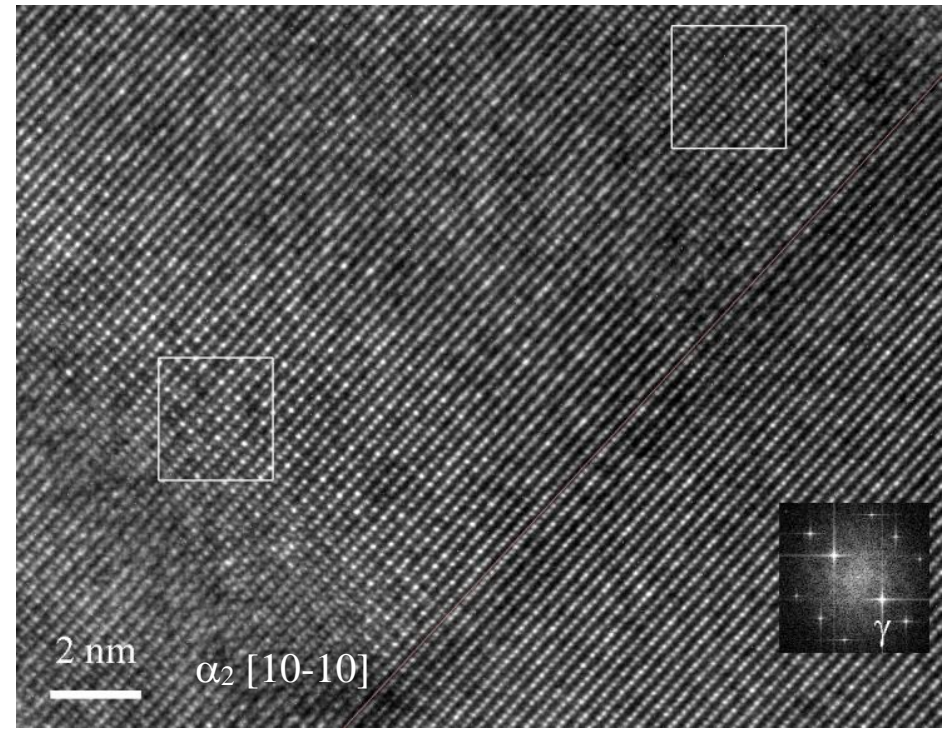

a.

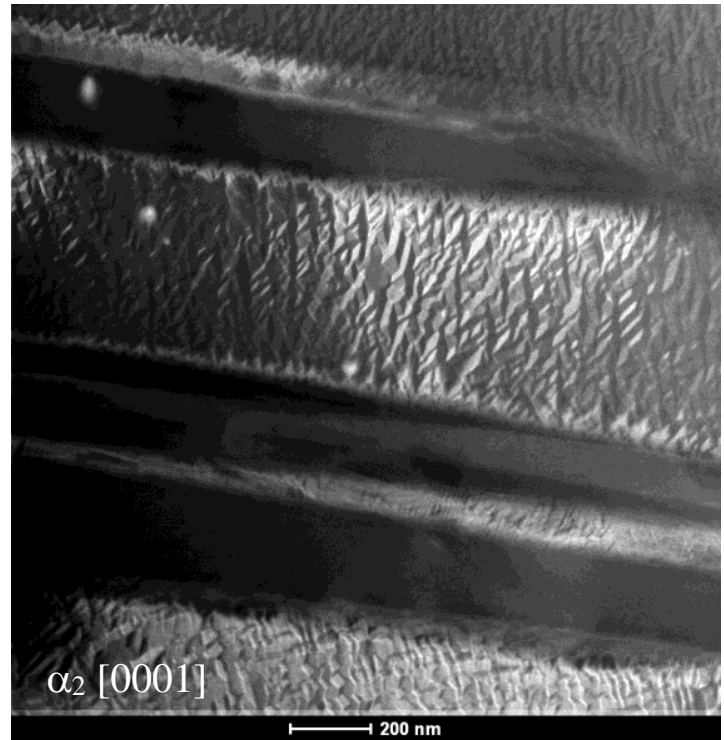

b.

Figure 2. a. Edge-on projection of interface between $\alpha_{2}$ lamella and neighboring $\gamma$ phase, 2 stripes of differently oriented O-phase can be resolved (see insets). b. [0001] projection of $\left(\alpha_{2}+\gamma\right)$ colony showing faceting in $\alpha_{2}$ lamellae. 